\title{
Socio-economic status, self-rated health and mental health: the mediation effect of social participation on early-late midlife and older adults
}

\author{
Netta Achdut ${ }^{*}$ (D) and Orly Sarid
}

\begin{abstract}
Background: Socioeconomic status (SES) is a major determinant of diverse health outcomes, among these are selfrated-health and mental health. Yet the mechanisms underlying the SES-health relation are not fully explored. Socioeconomic inequalities in health and mental health may form along several pathways. One is social participation which is linked to better self-rated-health and mental health. We examined (1) whether various social participation practices, including the usage of information and communication technology, relate to a unidimensional or multidimensional phenomenon (2) the relationship among SES, social participation, self-rated-health and mental health; (3) whether social participation and mental health mediates the association between SES and self-rated-health; (4) whether social participation and self-rated-health mediates the links between SES and mental health.
\end{abstract}

Method: Cross-sectional data for individuals aged 35 and older were taken from the Israeli Social Survey for $2016(N=4848)$. Social participation practices included connection with family and friends, self-perceived-support, self-perceived trust, volunteering, civic and political involvement, and information and communication technology usage. An exploratory factor analysis was conducted for all social participation practices. We then constructed structural Equation Modeling (SEM) to explore paths of relations among SES, social participation, self-rated-health and mental health.

Results: We found disparities in self-rated health and mental health across SES. Social participation practice, 'frequency of meeting with friends', mediated the links between SES-self-rated health and SES-mental health. Formal social participation practices along with internet usage mediated the SES- self-rated health link. Informal social participation practices and self-perceived trust mediated the SES-mental health link. Mental health mediated the SESself-rated health link and self-rated health mediated the SES-mental health link.

Conclusion: The links between SES and the two health constructs were enhanced by common and distinct social participation practices. Enhancement of social participation practices among low SES individuals is recommended. Social participation should be a prominent aspect of preventive medicine practice and health promotion interventions. Policy makers are called to support such programs as an important way to promote public health.

Keywords: Socio-economic status, self-rated-health, Mental health, Social participation, Information and communication technology, Structural equation models

\footnotetext{
* Correspondence: netta_ach@hotmail.com

The Spitzer Department of Social Work, Ben-Gurion University of the Negev,

84105 Beer-Sheva, Israe
}

(c) The Author(s). 2020 Open Access This article is distributed under the terms of the Creative Commons Attribution 4.0 International License (http://creativecommons.org/licenses/by/4.0/), which permits unrestricted use, distribution, and reproduction in any medium, provided you give appropriate credit to the original author(s) and the source, provide a link to the Creative Commons license, and indicate if changes were made. The Creative Commons Public Domain Dedication waiver (http://creativecommons.org/publicdomain/zero/1.0/) applies to the data made available in this article, unless otherwise stated. 


\section{Introduction}

Socioeconomic inequalities in health are an important topic in social sciences and public health. Since the Black Report of 1980, which established the structural explanation for health inequality over competing explanations [1], considerable effort has been invested to understand what causes these disparities, so as to be better able to identify measures to reduce them [2]. According to the structural explanation, health inequalities are the unjust differences in health between groups of people occupying different positions in society. That is, differences in the socioeconomic circumstances of social groups along the lifecourse cause differences in health outcomes. Structural theorists view competing explanations for health disparities as mechanisms linking structural determinants and health outcomes. Accordingly, the Black Report claimed that the causal explanation of health disparities is rooted in socioeconomic inequalities $[2,3]$.

Socioeconomic inequalities in health and mental health may form along several pathways [4]. One is social participation (SP), which is broadly defined as the individual's involvement in activities that include interactions with others in the society [5] and embraces various practices of informal and formal social connections and activities [6]. SP may be beneficial to health due to the support function provided to individuals by social networks through access to information, knowledge and to material and psychological resources that can be used to cope in times of need. SP also provides a platform for fulfilling an individual's needs for the social interaction and integration necessary for well-being.

Many previous studies that focused on the link between SP and health measures relied on samples of old adults [7-9]. Only few focused on younger ages or examined the mediating role of different SP practices in the SES-health association [10, 11]. Based on a wide range of SP practices, including information and communication technology (ICT), the current study investigated the mediating role of SP on the SES-self-rated health $(\mathrm{SRH})$ relationship and the mediating role of SP in the SES-mental health $(\mathrm{MH})$ connection in individuals aged 35 and above. In line with the Black Report's statement that differences in SES are the root cause of health disparities across the life span, it is important to better understand the factors potentially underlying health inequality in the general population. Few previous studies on this issue have taken this approach, including in their analysis both midlife and older adults $[6,12]$.

\section{Theoretical framework}

Social participation, previously conceptualized as social capital, refers to a broad aspect of support networks and the relationships within and between units such as family, social groups and neighborhood [13]. Putnam [14] argues that "the core idea of social capital is that social networks have a value. Social contacts affect the productivity of individuals and groups" (pp. 18-19). Social capital can thus be defined as a personal resource accessed through social relationships [6]. However, social capital is a complex construct with distinct components [14].

Berkman et al. [15] suggested a pathways model in which social structural conditions, such as SES and poverty, condition the extent, shape and nature of social networks, such as the characteristics of network ties. These ties, in turn, provide opportunities for social support, social engagement, and access to resources such as material goods and information. The benefits and gains from social networks eventually influence an individual's health. Berkman thus implies that individuals with low SES have weaker social networks than individuals with high SES and a lower degree of social participation. Possibly, low SES individuals struggle to provide for themselves and are less available to take part in various SP practices. Similarly, their peers and families are often struggling with their own daily needs and are not available to provide them with material, instrumental, or mental support [16]. That is, individuals of low SES are at a higher risk of experiencing weaker social support, a lower degree of a sense of belonging, and a limited access to information, whereas individuals of higher SES have greater resources and gains. Thus, SP may serve as an underlying mechanism in the SES - health link.

SP practices must, however, be differentiated. Berkman et al. [15] imply that not all ties are supportive and that the type, frequency, intensity, and extent of support provided vary across different social networks. Different SP practices involve diverse degrees of involvement and closeness and, hence, offer diverse rewards, some providing several types of support while other forms provide only one type or other human needs. For example, connections with family and friends are an important source of emotional and instrumental support. Other practices relating to social interactions outside the family include volunteer activity, membership in organizations and political involvement. They tend to build up civic skills, create an 'equivalent status and power' among their members and provide benefits such as information exchange and group identity. These SP practices are also called 'opportunity-based mechanisms' [6].

More recent conceptualization refers to SP practices by formal and informal social ties: formal SP comprises rule-bound networks whereas, informal SP is characterized by casual contacts with family and friends $[6,17]$.

The various SP practices might not uniformly affect health [18] let alone different construct of health, as $\mathrm{SRH}$ and $\mathrm{MH}$. In the digital age, Information and Communication Technology (ICT) is a central platform 
through which social connections are made and communities built. Digital platforms are not limited to social networks but also serve other important aspects of human life, such as seeking and exchanging information, improving job matching, obtaining services from government (e-government) more efficiently, including healthcare services [19], sharing health-related insights online, appropriateness of health service use [20] and easier take-up of social rights, such as social security benefits. Digital skills and actual usage are thus essential for participation in the digital economy and society. Services in the areas of health care and e-government, which are increasingly reliant on digitalized systems, may have a great effect on an individual's health and wellbeing [19].

Internet use may also reinforce offline relationships through computer-mediated communication and may increase offline contact and social capital [19]. On the other hand, the internet exposes people to new health risks by providing inaccurate or misleading information, by online reinforcement of pathologies, and by increasing confusion due to information overload [21, 22]. Intense internet use may also shift social interactions from the real to the virtual world, replacing social activity and strong ties [19].

\section{Previous studies}

SES is a major determinant of diverse health outcomes around the globe and across ages [23-26]. Previous studies showed that low socioeconomic status (SES) is related to higher mortality [27], poor self-rated health $(\mathrm{SRH})$ and mental health $(\mathrm{MH})[23,25,26,28]$, higher prevalence of obesity [28] and depression [24, 29].

A large body of empirical literature documents the contribution of SP to various health measures. Overall, a positive effect of SP on different health measures was reported, including reduced mortality [30], depression, loneliness and enhanced wellbeing $[9,11,29,31]$ and SRH $[9,32,33]$. Based on longitudinal data Giordano, Björk \& Lindström [34] examined the effect on SRH of social trust, frequency of meeting with friends, frequency of talking with neighbors, and being active in voluntary community groups, organizations or leisure group activities. Low levels of trust and talking less with neighbors preceded a gradual change from a good SRH to poor SRH baseline. These factors, along with the frequency of meeting with friends, also predicted improvement of SRH over time. Windsor et al. [35] employed four measures of social network structure relating to connection with family and friends (number, frequency of meeting, and duration of contact when meeting) along with participation in organized group activities. They also measured the social network quality by frequency of positive exchanges (expressions of care and interest) and negative exchanges (demands, criticisms, and arguments/tensions) in the domains of family and friend relationships. Participants with more diverse networks reported better mental health, showing direct associations of positive exchanges with better mental health, and negative exchanges with worse mental health. Using similar measures of SP, excluding family relations, Vogelsang [18] found that some aspects of SP are important for SRH, among them church or worship, meeting with friends, and charity and welfare activities. Other studies demonstrated that the link between SP and health measures varies with the form of SP and across different subgroups like gender, age and ruralurban contexts $[6,11,18,36]$. We assume that the gains from distinct SP practices differ by the degree and type of support and that the effect of distinct SP practices on health measures will also vary.

Evidence relating Internet usage to health is quite mixed. ICT usage has generally been found to be beneficial for various health outcomes among older adults, but less positive for adolescents and young adults [37-39]. For example, Internet use is associated with higher perceived health, reduced stress, less physical illness, and lower use of health services among adults independent of socio-demographic covariates [20,40]. However, in some studies this significant relationship disappeared once social class was considered [41]. Internet use was also positively associated with mental well-being among adults and older adults and with reduced probability of depression [42]. The recent OECD study reported that countries with a high level of internet use showed fewer reports of loneliness and Internet usage was positively correlated with general life satisfaction [19]. Yet, other studies found negative effects of Internet use, particularly the use of social networking sites, on various psychological well-being measures among adults $[43,44]$.

\section{The mediating role of social participation in the SES-health link}

Most studies have considered the direct effects of SES $[25,45,46]$, SP $(9,34,45,46)$, and ICT usage $[20,38]$ on $\mathrm{SRH}$ and $\mathrm{MH}$. Only few studies have examined the mediating role of SP practices on the relationship between SES and health. These studies employed single or few measures of SP as mediators, such as social trust [47] and family social capital [48]. Niedzwiedz et al. [11] showed that SP, measured by participation in external social activities (voluntary/charity, education and training courses, political or community organization) moderated the association between household wealth and loneliness among older adults. That is, participation in external social activities acts as a buffer against the adverse effects of socioeconomic disadvantage on loneliness. Rözer and Volker [47] found that social trust mediated the relation between income inequality and SRH, whereas other studies found no such effect [4]. But, as far as we know, no study to date has examined the role of the various SP practices suggested here as 
intermediary factors in the connection between SESSRH and SES-MH. We therefore explored the differential mediation effect of SP practices on the links between SES-SRH and SES-MH, controlling each measure of health in turn.

Our first aim here was to assess whether the various SP practices including ICT usage relate to a unidimensional world of content or reflect a multidimensional phenomenon. Our second aim was to test the relation among SES, SP, SRH and MH. We hypothesize that individuals of low SES will demonstrate lower SP and poorer SRH and MH.

Since several researchers have demonstrated the direct positive effect of SRH and other objective health measures on MH $[35,46,49,50]$, our third aim was to assess the contribution of $\mathrm{MH}$ on SES-SRH and the role of SRH on SES-MH. We hypothesize that MH mediates the SES-SRH link and that SRH mediates the SES-MH link. Our fourth aim was to examine which SP practices mediate the association between SES-SRH and which the association between SES-MH. We assumed that the SES-SRH and SES-MH links are mediated by common and distinct SP practices.

\section{Method}

\section{Source of data}

Cross sectional data were drawn from the Israeli Social Survey (ISS) for the year 2016 (Public-Use-File) conducted by the Israeli Central Bureau of Statistics (ICBS) [51]. The ISS comprises a representative sample of 7500 persons aged 20 and older of the permanent noninstitutional population of Israel, as well as residents of non-custodial institutions. Inclusion criteria in this study was individuals aged 35 and older, which yielded a total of 4848 respondents.

Self-report questionnaires, based on Blaise software developed by Statistics Netherlands [52] were administered by ICBS interviewers using laptops. The interviews were conducted in Hebrew, Arabic and Russian and each lasted about an hour [51].

\section{Measurement}

\section{Dependent variables}

Self-rated health (SRH) was measured by three items: (1) general health condition: "How is your health, overall?" The response options lay on a four-point Likert scale (very $\operatorname{good}(=0)$; $\operatorname{good}(=1)$; not so $\operatorname{good}(=2)$; not good at all $(=$ $3)$ ); (2) having any health or physical problem that interfered with daily functioning. Respondent were classified to three categories: no health or physical problem $(=0)$; health problem that did not interfere so much or did not interfere at all with daily functioning $(=1)$; health problem that greatly interfere or interfered with daily functioning (=2). (3) Had a specific health problem that might interfere with the daily function, such as $a$. walking or climbing stairs; $b$. getting dressed or bathing; $c$. carrying out household activities; $d$. problems with memory or concentration; $e$. vision problems; $f$. difficulty hearing. Responses were arrayed on a four-point Likert scale (no difficulty $(=0)$ to complete incapacity $(=3))$. The response options were than dichotomized to reflect no or slight difficulty $(=0)$ and significant or complete incapacity $(=1)$. These six dichotomized variables were summed up to an overall index, ranging from 0 to 6 . The index thus indicated the number of limitations that interfered with daily functioning. Finally, using the sum of the final scores in the three items detailed above, we created an overall physical health scale, ranging from 0 to 11 . We recoded the scale so that higher score reflected better self-rated health condition. Reliability analysis (Cronbach's $\alpha$ ) for this scale was not calculated. Following Taber [53] we did not assume that our measurement of health was unidimensional or that individuals who reported being in poor or bad health would suffer from several health problems. For example, an individual with memory or concentration limitation is not necessarily assumed to have additional health problems [53].

Mental health (MH) was assessed on six questions: (1) Do you ever feel lonely (frequently, sometimes, seldom, never)? (2) Have you felt pressured in the past 12 months? (3) Have you felt depressed in the past 12 months? (4) Did you feel able to deal with your problems in the past 12 months? (5) Did you feel full of energy in the last 12 months? (6) Have worries prevented you from sleeping in the last 12 months? Answers to items 2-6 were set on a four-point Likert scale from always $(=1)$ to never $(=4)$. After reversal of the questions (4) and (5), a summed score for these six questions was calculated. The final score ranged from 6 to 24, with a higher score reflecting better mental health. Cronbach's $\alpha=.769$.

The two measures SRH and MH correlated moderately. Pearson correlation coefficients were 0.41. Despite some overlap in these variables we treated them as separate constructs, [see also 9, 23].

\section{Independent variables}

Socio-Economic Status (SES) was measured by three indicators: education level, average income per capita in the household and home ownership (no home ownership (= 0 ); owns a home $(=1)$ ). Participants' highest education level was recorded according to the International Standard Classification of Education [54] and divided into low (less than lower secondary education, or lower secondary education completed), medium (upper secondary education or post-secondary non-tertiary education completed) and high (tertiary education completed). Average income per capita was defined by three categories: low 
(up to NIS 2000/USD 530); medium (NIS 2001/USD 531 - NIS 4000/USD 1060); and high (NIS 4001/USD 1061 and above).

\section{Mediating variables}

Social Participation (SP) was divided into informal and formal practices:

(1) Informal practices consisted of four questions: $a$. satisfaction with relations with family members (not so satisfied and not satisfied at all $(=0)$; very satisfied or satisfied $(=1)) ; b$. frequency of meeting friends, or talking to them. Measured on a fourpoint Likert scale (less than once a month $(=0)$; once or twice a month $(=1)$; once or twice a week $(=2)$; daily, or almost daily $(=3))$; $c$. "If you were in trouble, are there people whose help you could count on?" defined as self-perceived support $(1=$ yes); and $d$. "In general, can you trust most people or do you have to be wary of them?" defined as perceived trust $(1=$ most people can be trusted)

(2) Formal practices of SP included: $a$. engagement in volunteer activities in the previous 12 months, in the framework of an organization or movement $(=1) ; b$. involvement in public or political life on a national or local level in the past 12 months $(=1)$. Those who reported involvement in at least one activity received the value 1 .

An additional domain of SP was using Information and Communication Technology (ICT), measured by nine items signifying the intensity and purposes of this activity. The first item was: "During the last three months, how many times a week did you use the internet, through a computer or a mobile phone? $(0=$ not at all; $1=$ twice a week or less; $2=$ every day or almost every day). The remaining eight items were: used the internet $a$. to search for information; $b$. for e-mail; for discussion groups and social networks, such as chat rooms, forms, WhatsApp, Twitter; $c$. for online games or download games; $d$. for banking transactions and paying bills; $e$. for viewing or downloading files, such as photos, music, movies; $f$. to purchase products or services; $g$. to obtain services from government agencies; $h$. for phone calls or video, such as skype. Answers to each of the above items were dichotomies (yes $=1$ ). Based on the sum of these nine items we computed an ICT scale from 0 to 10 , a higher score reflecting more intense ICT use. Cronbach's $\alpha=.910$.

\section{Participants}

Table 1 summarizes the descriptive statistics of the sample. Age was fairly evenly distributed across the study population. The majority were married $(74 \%)$, with $44 \%$ having at least one child aged under 18 in the
Table 1 Descriptive statistics of the study variables, Israeli social survey for 2016

\begin{tabular}{|c|c|c|}
\hline & N & $\begin{array}{l}\text { \%/ Mean } \\
(\mathrm{SD})\end{array}$ \\
\hline \multicolumn{3}{|l|}{ Demographic } \\
\hline \multicolumn{3}{|l|}{ Age group } \\
\hline $35-44$ & 1377 & 28.4 \\
\hline $45-54$ & 1144 & 23.6 \\
\hline $55-64$ & 987 & 20.4 \\
\hline 65 and above & 1340 & 27.6 \\
\hline Male $(=1)$ & 2280 & 47.0 \\
\hline \multicolumn{3}{|l|}{ Marital status } \\
\hline Single & 326 & 6.7 \\
\hline Married & 3600 & 74.3 \\
\hline Divorced/Separated & 534 & 11.0 \\
\hline Widowed & 388 & 8.0 \\
\hline Children aged 0-17 in the household $(=1)$ & 2135 & 44.0 \\
\hline Immigrant (=1) & 914 & 18.9 \\
\hline Arab $(=1)$ & 876 & 18.1 \\
\hline Employed (=1) & 3004 & 62.0 \\
\hline \multicolumn{3}{|l|}{ SES } \\
\hline \multicolumn{3}{|l|}{ Education } \\
\hline Low & 1458 & 30.1 \\
\hline Medium & 1725 & 35.6 \\
\hline High & 1658 & 34.2 \\
\hline \multicolumn{3}{|l|}{ Average income per capita in the household } \\
\hline Low & 857 & 21.1 \\
\hline Medium & 1204 & 29.6 \\
\hline High & 2002 & 49.3 \\
\hline Home ownership (=1) & 3751 & 77.4 \\
\hline \multicolumn{3}{|l|}{ Social participation } \\
\hline $\begin{array}{l}\text { Satisfaction with family relationships ( } 1 \text { = very } \\
\text { satisfied or satisfied) }\end{array}$ & 4606 & 95.0 \\
\hline \multicolumn{3}{|l|}{ Frequency of meeting/speaking with friends (0-4) } \\
\hline No friends & 479 & 9.9 \\
\hline Less than once a month & 125 & 2.6 \\
\hline Once or twice a month & 521 & 10.8 \\
\hline Once or twice a week & 1815 & 37.6 \\
\hline Daily, or almost daily & 1889 & 39.1 \\
\hline Self-perceived-support (yes = 1) & 4371 & 91.3 \\
\hline Self-perceived-trust (yes $=1$ ) & 1925 & 41.2 \\
\hline Volunteering $(=1)$ & 951 & 19.6 \\
\hline Public/political involvement (=1) & 637 & 13.1 \\
\hline ICT use scale (0-10) & 4838 & $6.11(4.11)$ \\
\hline$N$ & 4848 & \\
\hline
\end{tabular}


household. Immigrants and the Arab respondents each comprised almost one fifth of the sample (18.9 and $18.1 \%$, respectively). Sixty-two percent were employed, where this rate was higher among the working-age population (77.3\%) (not shown). As for SES indicators, medium or high education was reported by 34.2 and $35.6 \%$ of the respondents respectively, as compared with a lower rate of respondents with low education (30.1\%). About half of the respondents had high average income per capita, and more than three-quarters reported home ownership.

The great majority of respondents (95\%) reported being very satisfied or satisfied with regard to family relations, about $77 \%$ stated that they met or spoke with friends once a week or more, and about $90 \%$ had people to count on in times of trouble. By contrast, when referring to self-perceived trust, only $41 \%$ believed that most people could be trusted. About $20 \%$ had taken part in volunteering activity, and the mean level of use of ICT was rather high, at 6.11 (on a $0-10$ scale).

\section{Data analysis}

For the various SES indicators, we conducted Varimax exploratory factor analysis (EFA) [55] using the initial Eigenvalue cutoffs to estimate the factor analysis. The factor solution yielded one factor with Eigenvalue above 1.0, comprising two indicators that loaded significantly: education level (.823), and average income per-capita in the household (.823). The cumulative variance explained by this factor was $67 \%$. Home ownership did not correlate closely with the other components, therefore we treated it as a distinct variable.

To examine the first aim, we conducted Varimax exploratory factor analysis (EFA) for all forms of SP. Two factors emerged with Eigenvalue above 1.0, the first comprised of three items that loaded significantly: volunteering (.675), political involvement (.725) and use of ICT (.671); the second was satisfaction with relationships with family members (.783) and self-perceived-support (.704). The cumulative variance explained by these two factors stood at $53 \%$. Two distinct items did not correlate with the above two factors: 'frequency of meeting friends or talking with them' and 'self-perceived-trust'.

To test the second aim, we conducted bivariate analysis of SRH and MH by SES and by SP. The Pearson correlation was calculated for SES factor and SRH, for SP factors and SRH, and for ICT scale and SRH. The same was done with MH. Using ANOVA (F-test) we examined mean differences in SRH and MH by the categorical variables of education, household income, and frequency of meeting with friends. The t-test examined mean differences in SRH and MH by home ownership, volunteering, political/civic involvement, satisfaction with family relations, perceived support and perceived trust. Six hierarchical linear regression models - three for each health outcome - estimated SRH and MH (results from these estimations are presented in Additional file 1:

To investigate the third and fourth aims, we constructed two Structural Equation Models (SEM) using SPSS statistic 25 with AMOS module. Both models were drawn by placing demographic variables detailed in Table 1 (not shown in the final SEM models) and SES indicators as predictors for SRH and MH. Next, by an iterative process, for each model we added pathways that improved the model's fit. Given a postulated association between the SP forms we also drew residual covariance paths between these variables. Adding these paths and the residual covariance paths was supported by the AMOS modification indices function. All models were estimated by the maximum likelihood estimation method. Since quality of the fit of SEM models is affected by sample size, multiple model fit indicators were assessed including $X^{2}$, the ratio of the $X^{2}$ to degrees of freedom $\left(x^{2} / \mathrm{df}\right)$, the comparative fit index (CFI), the Tucker-Lewis Index (TLI), the Normed Fit Index (NFI) and a root-mean-square error of approximation (RMSEA). Ideally, for a model that fitted the data, the $x^{2}$ would not be significant. However, since $\chi^{2}$ is sensitive to sample size, in cases of large samples, it has been suggested to use the ratio of the $x^{2}$ to degree of freedom. A model demonstrates a reasonable fit if the statistic adjusted by its degrees of freedom is $<5.0$ and $>2[56,57]$. Values close to 0.95 for the CFI, TLI and NFI (higher is better), and close to 0.05 for the RMSEA (lower is better) indicate a good fit of the data to the model [58].

Bootstrapping was used to test the significance of the mediation effect. This is a non-parametric method based on resampling with replacement, which is reiterated many times within the study sample according to its original size [59]. The indirect effect is computed from each of these samples and a sampling distribution can be empirically generated. The distribution allows computing a confidence interval (CIs), which is checked to determine if zero is in the interval. If zero is not in the interval, then the researcher can be confident that the indirect effect is different from zero, i.e., statistically significant [60]. We further tested the robustness of our results on the mediation effect using the RMediation package, which also builds CIs for mediation effects based on the product of two regression coefficients and their standard error [61]. Based on the CIs we tested the significance of each specific mediation. As in bootstrapping, in cases when zero is not in the $95 \%$ confidence interval, it can be concluded that the indirect effect is significantly different from zero at $p<.05$. 


\section{Results}

\section{Descriptive results}

Table 2 presents the mean value (SD) of SRH and $\mathrm{MH}$ indices by SES and SP. With respect to continuous explanatory variables, Pearson correlations are presented.

The top panel of Table 2 shows the mean value of $\mathrm{SRH}(8.23 \pm 3.10)$ and $\mathrm{MH}(18.04 \pm 3.94)$ in the overall sample, indicating relatively high values. Both $\mathrm{SRH}$ and $\mathrm{MH}$ mean values differed on all independent variables and indices. As expected, a positive correlation was found between the SES factor to SRH and $\mathrm{MH}$, and between home ownership to SRH and MH. SP was associated with better $\mathrm{SRH}$ and $\mathrm{MH}$ : factor 1 (volunteering, political and ICT use) correlated with SRH, whereas factor 2 ('satisfaction with family relations' ${ }^{1}$ and 'having people to count on') correlated with $\mathrm{MH}$.

\section{SEM models}

Figures 1 and 2 present two estimated SEM models for $\mathrm{SRH}$ and $\mathrm{MH}$, respectively. Standardized coefficients, based on bootstrapping, are displayed on the figures. Additionally, Additional file 1 includes full regression results based on OLS estimations for SRH and MH. We present only the mediation effects that were statistically significant based on the RMediation test detailed in data analysis section. SRH (Fig. 1): the $\chi^{2}$ value was significant in this model $\left(\chi^{2}(15)=58.02, p>0.05\right)$ due to the large sample size $(n=4848)$. However, this model gave a very good fit in all other indices $\left(\chi^{2} / \mathrm{df}=3.41\right.$, RMSEA $=.024, \mathrm{CFI}=$ .995 , TLI $=.984, \mathrm{NFI}=.993)$, and explained $42.2 \%$ of SRH variances. One direct pathway linked the SES factor to $\mathrm{SRH}$ (standardized regression weights $\beta=.18, p>0.01$ ). Three mediating pathways appear in the model linking SES to SRH: in the first mediating pathway, $\mathrm{MH}$ mediated the links between SES to SRH. To elaborate, higher SES led to better $\mathrm{MH}(\beta=.19, p>0.01)$ which in turn led to better SRH $(\beta=.36, p<0.001)$. The second indirect pathway indicated that the formal SP factor of volunteering, political and civic involvement and ICT mediated the links between SES and better SRH. That is, higher SES produced a higher score in this factor $(\beta=.386, p<0.001)$, which led to better SRH $(\beta=.50, p<0.01)$. In the third mediating pathway, 'frequency of meeting friends' served as a mediator between SES to SRH: SES impacted friends' relations $(\beta=.14, p<0.001)$ and friends' relations enhanced SRH $(\beta=.04, p<0.01)$.

$\mathrm{MH}$ (Fig. 2): the $\chi^{2}$ value was significant in this model $\left(\chi^{2}(18)=55.37, p>0.05\right)$. However, this model gave a very good fit for all other indices $\left(x^{2} \quad / d f=3.07\right.$,

\footnotetext{
${ }^{1}$ No substantial variance was found in the variable satisfaction with family relationship (see Table 1). However, this measure is part of the factor score of informal SP. Being part of a total factor score somewhat reduces this problem.
}

RMSEA $=.021, \mathrm{CFI}=.995, \mathrm{TLI}=.983, \mathrm{NFI}=.993)$, and addressed $30.0 \%$ of $\mathrm{MH}$ variance. Direct pathways linked home ownership with $\mathrm{MH}(\beta=.06, p<0.05)$, but no direct pathway was found between SES and MH $(\beta=.025$, n.s). Results from OLS regressions showed that once SP indicators were added as predictor, the SES coefficient became insignificant in explaining $\mathrm{MH}$ (see Additional file 1: , model 4-6). This suggests that there was full mediation. Fig. 2 reveals four pathways from SES to MH. In one pathway SRH mediated the links between SES and $\mathrm{MH}$. In another pathway the SP practice of 'family relations and self-perceived-support' mediated the links between SES and MH. The SES-MH pathway was also mediated by self-perceived-trust. In another pathway SES-MH was mediated by the practice 'frequency of meeting friends'.

\section{Discussion}

Since the 1980 Black Report, establishing the structural explanation for health inequalities [1], considerable effort has been exerted to understand what causes this inequality, so as to be better able to identify measures to reduce it [2]. In this study we proposed an optional pathway to the SES-health link through a measure of diverse SP practices along with Internet use.

Our findings confirm that SP is a multidimensional construct $[14,18]$ and are supported, to some extent, by the conceptualization of SP practices as formal and informal forms. Informal SP forms comprise family relations and perceived support, whereas formal SP practices include volunteering, political and civic involvement and ICT usage. The 'frequency of meeting friends' and perceived trust were distinct SP practices that did not align with the formal or informal forms of SP. Although ICT usage potentially embraces informal practices such as casual connection with close and remote friends and relatives through Facebook, WhatsApp or other social networks, it can be used as a platform for formal practices through membership in organizations, activity in social and civic movements and community activity through a neighborhood Facebook page. ICT, as measured here, refers also to uses other than SP that have proven health benefit $[19,20]$. For example, information searches and the use of e-services facilitate accumulation of knowledge and access to health services and treatment options. Either way, the classification of Internet use as part of formal SP practices needs to be further examined in future studies.

As we hypothesized, we found disparities in SRH and $\mathrm{MH}$ across SES. These findings here fit the accumulated knowledge on the relations between SES-SRH and SES$\mathrm{MH}$ [23-25]. Here SRH was a predictor of $\mathrm{MH}$ and vice versa as shown by the OLS estimations. We confirm previous studies showing that $\mathrm{MH}$ and others aspects of 
Table 2 SRH and MH by SES and social participation, Israeli social survey for 2016

\begin{tabular}{|c|c|c|c|c|}
\hline & $\begin{array}{l}\text { SRH Mean } \\
\text { (SD) }\end{array}$ & $\begin{array}{l}\text { F/ T-test/Pearson } \\
\text { correlation }\end{array}$ & $\begin{array}{l}\text { MH Mean } \\
(\mathrm{SD})\end{array}$ & $\begin{array}{l}\text { F/ T-test/Pearson } \\
\text { correlation }\end{array}$ \\
\hline Overall & $8.23(3.10)$ & & $18.04(3.94)$ & \\
\hline \multicolumn{5}{|l|}{ SES } \\
\hline \multicolumn{5}{|l|}{ Education } \\
\hline Low & $6.89(3.60)$ & $231.34^{* *}$ & $16.97(4.45)$ & $76.11^{* *}$ \\
\hline Medium & $8.53(2.80)$ & & $18.45(3.85)$ & \\
\hline High & $9.11(2.46)$ & & $18.54(3.35)$ & \\
\hline \multicolumn{5}{|l|}{ Average income per capita in the household } \\
\hline Low & $7.16(3.68)$ & $119.58^{* *}$ & $16.81(4.48)$ & $62.81^{* *}$ \\
\hline Medium & $7.73(3.32)$ & & $17.85(4.12)$ & \\
\hline High & $8.91(2.50)$ & & $18.59(3.42)$ & \\
\hline \multicolumn{5}{|l|}{ Home ownership } \\
\hline Yes & $8.34(3.03)$ & $-4.51^{* *}$ & $18.31(3.82)$ & $-8.68^{* *}$ \\
\hline No & $7.86(3.31)$ & & $17.12(4.21)$ & \\
\hline SES Factor: education Average Income per capita in the household & - & $.313^{* *}$ & - & $.228^{* *}$ \\
\hline \multicolumn{5}{|l|}{ Social participation } \\
\hline \multicolumn{5}{|l|}{ Volunteering } \\
\hline Yes & $9.12(2.39)$ & -11.73 & $18.67(3.47)$ & $-5.93 * *$ \\
\hline No & $8.02(3.21)$ & & $17.89(4.03)$ & \\
\hline \multicolumn{5}{|l|}{ Public/political involvement } \\
\hline Yes & $8.11(3.18)$ & $-8.68^{* *}$ & $18.62(3.18)$ & $-4.65^{* *}$ \\
\hline No & $9.02(2.33)$ & & $17.95(4.04)$ & \\
\hline ICT use scale $(0-10)$ & & $.407^{* *}$ & $.157^{* *}$ & \\
\hline Factor 1: volunteering, political involvement, use of ICT & - & $.289^{* *}$ & - & $.125^{* *}$ \\
\hline \multicolumn{5}{|l|}{ Satisfaction with family relationships } \\
\hline Very satisfied or satisfied & $8.30(3.07)$ & $-5.36^{* *}$ & $18.18(3.84)$ & $-9.13^{* *}$ \\
\hline Not so satisfied or not satisfied at all & $7.00(3.41)$ & & $15.07(4.66)$ & \\
\hline \multicolumn{5}{|l|}{ Self-perceived-support } \\
\hline Yes & $8.39(3.01)$ & $-8.88^{* *}$ & $18.27(3.77)$ & $-10.47^{* *}$ \\
\hline No & $6.79(3.57)$ & & $15.70(4.67)$ & \\
\hline Factor 2: satisfaction with family relations, having people to count on in times of trouble & - & $.171^{* *}$ & - & $.221^{* *}$ \\
\hline Frequency of meeting/talking with friends (0-4) & - & $.208^{* *}$ & - & $.174^{* *}$ \\
\hline No friends & $6.35(3.69)$ & $57.11^{* *}$ & $15.93(4.80)$ & $42.12^{* *}$ \\
\hline Less than once a month & $7.45(3.43)$ & & $17.86(4.01)$ & \\
\hline Once or twice a month & $8.03(2.99)$ & & $17.60(4.04)$ & \\
\hline Once or twice a week & $8.39(3.04)$ & & $18.27(3.72)$ & \\
\hline Daily, or almost daily & $8.66(2.81)$ & & $18.46(3.71)$ & \\
\hline \multicolumn{5}{|l|}{ Self-perceived-trust } \\
\hline Most people you can trust & $8.85(2.72)$ & $-10.85^{* *}$ & $18.76(3.47)$ & $-10.432^{* *}$ \\
\hline You have to be wary of people & $7.87(3.25)$ & & $17.54(4.14)$ & \\
\hline
\end{tabular}

${ }^{*} p<.05 ;{ }^{* *} p<.01$

well-being, such as happiness, enjoyment and optimism, were positively related to SRH and physical health and negatively related to disease and mortality $[35,46,49$, 50]. Furthermore, as shown in our SEM models, better $\mathrm{MH}$ mediated the SES-SRH association, whereas SRH mediated the SES-MH link. These findings, along with those from other studies, demonstrate that health and mental health are two interrelated but different phenomena which have both common risk factors and distinct resilience factors [62, 63]. 


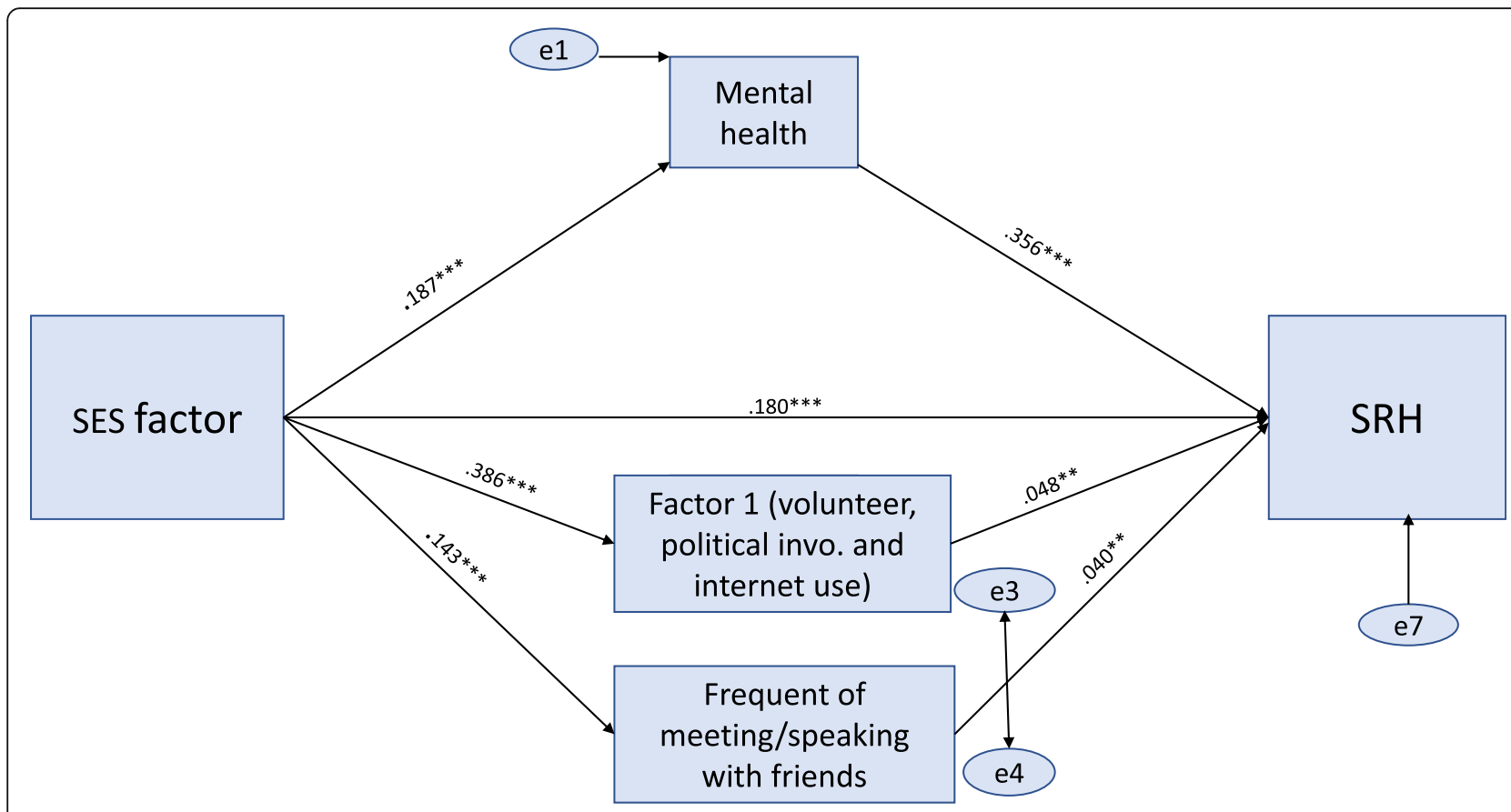

Note. ${ }^{* *} \mathrm{p}<.01 ; * * \mathrm{p}<.001$

Fig. 1 Path analysis model showing the direct and indirect effects of SES on SRH, with mental health and social participation as mediators

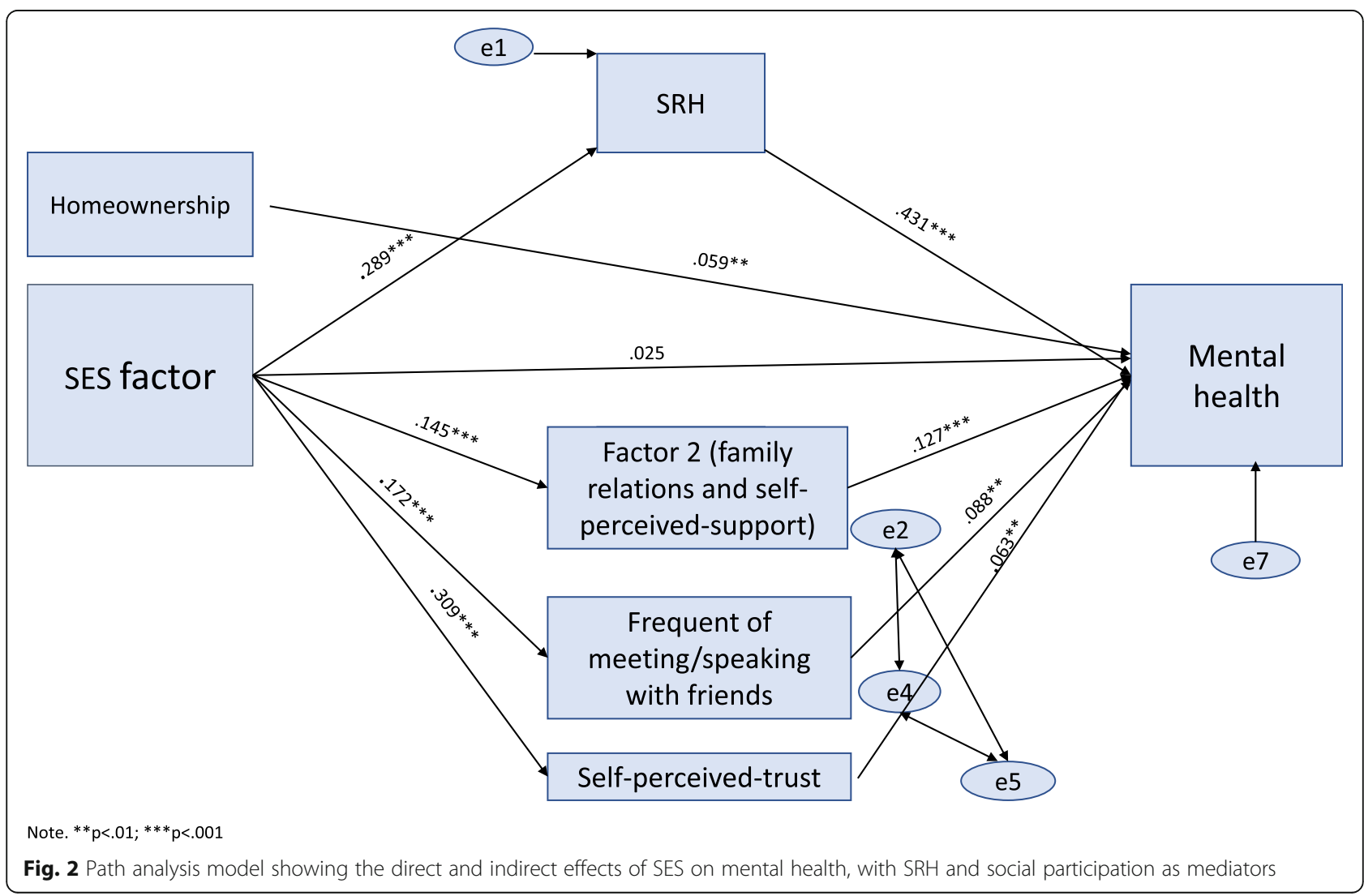


Finally, we examined the distinct mediating role of SP forms on SES-SRH and SES-MH assuming that SP is a multidimensional concept with different intensities of effects on health, as well as different constructs of health. Higher SES was associated with stronger SP, which, in turn, was positively related to both SRH and MH. To elaborate, health inequality was partly related to a lesser degree of both formal and informal SP practices. Low SES individuals may engage less in the various forms of social participation or benefit less from these interactions and activities, which might result in a worse health outcome. As we assumed, variation in SP mediators were found across the two health constructs. SRH was enhanced by formal SP forms including Internet use practice while the SES-MH link was mediated by informal SP forms and self-perceived-trust. Our results demonstrate that different SP gains are important in enhancing different health outcomes. A possible explanation for the mediating role of formal SP practices and ICT use in the SES-SRH link is that these practices enable individuals to access information, gather knowledge and power and exert a certain degree of control over their physical health. In the SES-MH link informal SP was important. Having social trust, a sense of support and a supportive network of family ties may imply close and intimate relationships that benefit mental health [15]. Furthermore, mediation effects also varied in intensity across the models: While informal SP practices, frequency of meeting with friends and trust fully mediated the SES-MH link, formal SP practices and frequency of meeting with friends only partially mediated the SES-SRH link. This may mean that "physical health", more than $\mathrm{MH}$, is influenced by "real" socio-economic circumstances, such as limited access to healthcare and insufficient nutrition. It also may imply that alternative underlying mechanisms are important in the SES-SRH link, such as risk behaviors and macro-level mechanisms, including the characteristics and the quality of the healthcare system and public health expenditure. However, keeping in mind that SP practices do not uniformly affect $\mathrm{SRH}$ and $\mathrm{MH}$, it is important to look for practices that can enhance both SRH and MH. Such practices can serve as a first line protective mechanism in enhancing diverse health constructs. In our SEM models a significant mediator was frequency of meeting with friends and thus regularity and constancy of meeting friends may be important in enhancing both SRH and $\mathrm{MH}$. Other regular practices that relate to better health are daily habits, and frequency of meeting friends can be seen as a habit showing recurrence and repetition important in promoting health.

This study has several limitations. Given its crosssectional design, our attributions of causality must be taken with caution. Although our explanations are rooted in theoretical models and research, and the SEMs suggest causality, alternative explanations cannot be ruled out, and plausible alternative models may fit the data as well or better than the models we tested. Our aim in this analysis was to help develop knowledge by examining potential underlying mechanism of the SEShealth link, an approach used by previous health studies also in examining potential mediators in the SES-health link $[4,23]$.

Since our tests show that SP mediators are significant, they provide a strong rationale for testing the causal link through longitudinal data or experimental designs. Thus, we recommend a big data base to adapt a longitudinal prospective design and to follow the same individuals across time. Furthermore, given the complexity of our model and analyses, we did not test alternative path directions (health-to-SES or SP-to-SES) or subgroup differences by age or ethno-culture groups. Future studies should address these issues. Finally, important mediators such as financial strain and deprivation indices should also be taken into account in exploring the paths between SES and health.

\section{Conclusions and recommendations}

Implication of this study are relevant for policy makers as well as for health and social services practitioners. The enhancement of SP practices should be considered as a way to promote health-in older adults, but also in early-late midlife adults. Particularly among lower SES individuals, enhancement of formal practices of SP, such as teaching ICT use, encouraging volunteering and community activities may improve their SRH. Practitioners working in the community and in mental health services are encouraged to collaborate and construct interventions to enhance perceived trust and informal SP forms, especially in low SES groups as these can act as buffers in the SES-MH association. Moreover, considering the aims of an intervention and the target population, interventions should place different emphases on specific SP practices. For example, while, frequent meetings with friends should be a central aspect in an SP intervention that aims to enhance both SRH and $\mathrm{MH}$, enhancing perceived trust should be a central aspect in the field of $\mathrm{MH}$. Finally, as suggested by both mediation models, interventions to promote $\mathrm{MH}$ may help to moderate SES's negative effect on SRH and vice versa. Policy makers should support programs enhancing SP as an important means of promoting public health, regardless of the need to deal with the root causes of health inequality in society, i.e., socioeconomic disparities.

\section{Supplementary information}

Supplementary information accompanies this paper at https://doi.org/10. 1186/s13584-019-0359-8.

Additional file 1: OLS Regression: prediction of $\mathrm{SRH}$ and mental health. 


\section{Abbreviations}

ICT: Information and communication technology; MH: Mental health; SES: Socioeconomic status; SP: Social participation; SRH: Self-rated-health

\section{Acknowledgements}

Not Applicable.

\section{Authors' contributions}

N.A. and O. S conceptualized the aims, conducted the statistical analyses, drafted the manuscript and worked on its final version. Both authors read and approved this final version. All authors read and approved the final manuscript.

\section{Funding}

This research did not receive any specific grant from funding agencies in the public, commercial, or not-for-profit sectors.

\section{Availability of data and materials}

The data sets analyzed during the current study are available from the Israel Central Bureau of Statistics upon request.

\section{Ethics approval and consent to participate}

We hereby declare that the data were collected by the Israel Central Bureau of Statistics, and are subject to that Bureau's Ethics Committee. Informed consent was obtained from all individual participants in the present study.

\section{Consent for publication}

Not Applicable.

\section{Competing interests}

The authors declare that they have no conflict of interests.

Received: 20 June 2019 Accepted: 23 December 2019

Published online: 28 January 2020

\section{References}

1. Black D, Morris JN, Smith C, Townsend P. Inequalities in health: the Black report. London: Penguin Books; 1982

2. McCartney G, Collins C, Mackenzie M. What (or who) causes health inequalities: theories, evidence and implications? Health Policy. 2013;113(2):221-227. https://doi.org/10.1016/j.healthpol.2013.05.021.

3. Macintyre S. The Black report and beyond what are the issues? Soc Sci Med. 1997:44(6):723-45. https://doi.org/10.1016/S0277-9536(96)00183-9.

4. Vilhjalmsdottir A, Gardarsdottir RB, Bernburg JG, Sigfusdottir ID. Neighborhood income inequality, social capital and emotional distress among adolescents: a population-based study. J Adolesc. 2016;51:92-102. https://doi.org/10.1016/j.adolescence.2016.06.004

5. Levasseur M, Richard L, Gauvin L, Raymond É. Inventory and analysis of definitions of social participation found in the aging literature: proposed taxonomy of social activities. Soc Sci Med. 2010;71(12):2141-9.

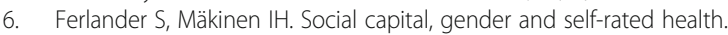
Evidence from the Moscow health survey 2004. Soc Sci Med. 2009;69(9): 1323-332. https://doi.org/10.1016/J.SOCSCIMED.2009.08.009.

7. Chen JH, Lauderdale DS, Waite LJ. Social participation and older adults' sleep. Soc Sci Med. 2016;149:164-73. https://doi.org/10.1016/J.SOCSCIMED 2015.11.045

8. Escobar-Bravo MÁ, Puga-González D, Martín-Baranera M. Protective effects of social networks on disability among older adults in Spain. Arch Gerontol Geriatr. 2012;54(1):109-116. https://doi.org/10.1016/J.ARCHGER.2011.01.008.

9. Gilmour $\mathrm{H}$. Social participation and the health and well-being of Canadian seniors. Health Rep. 2012;23(4):3-12

10. Vilhjalmsdottir A, Gardarsdottir RB, Bernburg JG, Sigfusdottir ID. Neighborhood income inequality, social capital and emotional distress among adolescents: a population-based study. J Adolesc. 2016;51:92-102.

11. Niedzwiedz CL, Richardson EA, Tunstall H, Shortt NK, Mitchell RJ, Pearce JR The relationship between wealth and loneliness among older people across Europe: is social participation protective? Prev Med. 2016;91(3):24-31. https://doi.org/10.1016/J.YPMED.2016.07.016

12. Ferreira-Alves J, Magalhães P, Viola L, Simoes R. Loneliness in middle and old age: demographics, perceived health, and social satisfaction as predictors. Arch Gerontol Geriatr. 2014;59(3);613-623. https://doi.org/10. 1016/J.ARCHGER.2014.06.010.

13. Coleman JS. Social capital in the creation of human capital. Am J Sociol. 1988:94:S95-S120. https://doi.org/10.1086/228943.

14. Putnam R. Bowling alone: the collapse and revival of American community. New York: Simon \& Schuster; 2000.

15. Berkman LF, Glass T, Brissette I, Seeman TE. From social integration to health: Durkheim in the new millennium. Soc Sci Med. 2000;51:843-457.

16. Desmond M. Disposable ties and the urban poor. Am J Sociol. 2012;117(5): 1295-335. https://doi.org/10.1086/663574

17. Adams KB, Leibbrandt $\mathrm{S}$, Moon $\mathrm{H}$. A critical review of the literature on social and leisure activity and wellbeing in later life. Ageing Soc. 2011;31(04):683712. https://doi.org/10.1017/S0144686X10001091.

18. Vogelsang EM. Older adult social participation and its relationship with health: rural-urban differences. Health Place. 2016;42(4):111-19. https://doi. org/10.1016/J.HEALTHPLACE.2016.09.010.

19. OECD. How's life in the digital age?: opportunities and risks of the digital transformation for People's well-being. Paris OECD Publishing. 2019. p 1312. Retrieved https://doi.org/10.1787/9789264311800-en.

20. Deetjen U, Powell JA. Internet use and health: connecting secondary data through spatial microsimulation. Digit Health. 2016;2:1-17. https:/doi.org/ $10.1177 / 2055207616666588$.

21. Rice RE. Influences, usage, and outcomes of internet health information searching: multivariate results from the pew surveys. Int J Med Inform. 2006:75(1):8-28. https://doi.org/10.1016/j.jjmedinf.2005.07.032.

22. Ziebland S, Wyke S. Health and illness in a connected world: how might sharing experiences on the internet affect people's health? Milbank Q. 2012; 90(2):219-49. https://doi.org/10.1111/j.1468-0009.2012.00662.x.

23. Meyer OL, Castro-Schilo L, Aguilar-Gaxiola S. Determinants of mental health and self-rated health: a model of socioeconomic status, neighborhood safety, and physical activity. Am J Public Health. 2014;104(9):1734-741 https://doi.org/10.2105/AJPH.2014.302003.

24. Domènech-Abella J, Mundó J, Leonardi M, Chatterji S, Tobiasz-Adamczyk B, Koskinen S, Haro JM. The association between socioeconomic status and depression among older adults in Finland, Poland and Spain: a comparative cross-sectional study of distinct measures and pathways. J Affect Disord. 2018;241:311-18. https://doi.org/10.1016/j.jad.2018.08.077.

25. Hu Y, van Lenthe FJ, Borsboom GJ, Looman CWN, Bopp M, Burström B Mackenbach JP. Trends in socioeconomic inequalities in self-assessed health in 17 European countries between 1990 and 2010. J Epidemiol Community Health. 2016;70(7):644-652. https://doi.org/10.1136/jech-2015-206780.

26. Reiss F. Socioeconomic inequalities and mental health problems in children and adolescents: a systematic review. Soc Sci Med. 2013;90:24-31. https:// doi.org/10.1016/J.SOCSCIMED.2013.04.026

27. Doubeni CA, Schootman M, Major JM, Stone RAT, Laiyemo AO, Park Y, Schatzkin A. Health status, neighborhood socioeconomic context, and premature mortality in the United States: the National Institutes of HealthAARP diet and health study. Am J Public Health. 2012;102(4):680-88. https:// doi.org/10.2105/AJPH.2011.300158.

28. Baum CL, Ruhm CJ. Age, socioeconomic status and obesity growth. J Health Econ. 2009;28(3):635-48. https://doi.org/10.1016/J.JHEALECO.2009. 01.004.

29. Everson SA, Maty SC, Lynch JW, Kaplan GA. Epidemiologic evidence for the relation between socioeconomic status and depression, obesity, and diabetes. J Psychosom Res. 2002:53(4):891-95. https://doi.org/10.1016/ S0022-3999(02)00303-3.

30. Folland S. Does "community social capital" contribute to population health? Soc Sci Med. 2007;64(2):2342-354. https://doi.org/10.1016/j.socscimed.2007. 03.003.

31. Valk I. Social capital as a solution to mental health problems: the effect of social capital on 'depression' and 'loneliness' via the effect of social cohesion and civic engagement. Utrecht University, Utrecht, The Netherlands: Unpublished master's thesis; 2017.

32. Ichida $Y$, Hirai $H$, Kondo $K$, Kawachi I, Takeda $T$, Endo $H$. Does social participation improve self-rated health in the older population? A quasiexperimental intervention study. Soc Sci Med. 2013:94(2):83-90. https://doi. org/10.1016/J.SOCSCIMED.2013.05.006.

33. Verhaeghe PP, Pattyn E, Bracke P, Verhaeghe M, Van De Putte B. The association between network social capital and self-rated health: pouring old wine in new bottles? Health Place. 2012;18(2):358-65. https://doi.org/10. 1016/J.HEALTHPLACE.2011.11.005. 
34. Giordano GN, Björk J, Lindström M. Social capital and self-rated health: a study of temporal (causal) relationships. Soc Sci Med. 2012;75(2):340-48. https://doi.org/10.1016/J.SOCSCIMED.2012.03.011.

35. Windsor TD, Rioseco P, Fiori KL, Curtis RG, Booth H. Structural and functional social network attributes moderate the association of self-rated health with mental health in midlife and older adults. Int Psychogeriatr. 2016;28(01):4961. https://doi.org/10.1017/S1041610215001143.

36. Lee H, Y, Jang SN, Lee S, Cho SI, Park EO. The relationship between social participation and self-rated health by sex and age: a cross-sectional survey. Int J Nurs Stud. 2008;45(7):1042-1054. https://doi.org/10.1016/J.IJNURSTU. 2007.05.007.

37. Kross E, Verduyn P, Demiralp E, Park J, Lee DS, Lin N, et al. Facebook use predicts declines in subjective well-being in young adults. PLoS One. 2013;8(8):e69841. https://doi.org/10.1371/journal.pone.0069841.

38. Panova T, Lleras A. Avoidance or boredom: negative mental health outcomes associated with use of information and communication technologies depend on users' motivations. Comput Hum Behav. 2016;58, 249-258. https://doi.org/10.1016/J.CHB.2015.12.062.

39. Thomée S, Härenstam A, Hagberg M. Mobile phone use and stress, sleep disturbances, and symptoms of depression among young adults - a prospective cohort study. BMC Public Health. 2011;11(1):66. https://doi.org/ 10.1186/1471-2458-11-66

40. Nabi RL, Prestin A, So J. Facebook friends with (health) benefits? Exploring social network site use and perceptions of social support, stress, and wellbeing. Cyberpsychol Behav Soc Netw. 2013;16:721-7.

41. Gracia E, Herrero J. Internet use and self-rated health among older people: a national survey. J Med Internet Res. 2009;11(4):e49. https://doi.org/10.2196/ jmir.1311.

42. Cotten SR, Ford G, Ford S, Hale TM. Internet use and depression among older adults. Comput Hum Behav. 2012;28(2):496-99. https://doi.org/10. 1016/J.CHB.2011.10.021.

43. Huang C. Internet use and psychological well-being: a meta-analysis. Cyberpsychol Behav Soc Netw. 2010;13(3):241-49. https://doi.org/10.1089/ cyber.2009.0217.

44. Sabatini F, Sarracino F. Online networks and subjective well-being. Kyklos. 2017;70(3):456-80

45. Kunst AE, Bos V, Lahelma E, Bartley M, Lissau I, Regidor E, Mackenbach JP. Trends in socioeconomic inequalities in self-assessed health in 10 European countries. Int J Epidemiol. 2005;34(2):295-305. https://doi.org/10.1093/ije/ dyh342.

46. Ahmad F, Jhajj AK, Stewart DE, Burghardt M, Bierman AS. Single item measures of self-rated mental health: a scoping review. BMC Health Serv Res. 2014;14(1):398. https://doi.org/10.1186/1472-6963-14-398.

47. Rözer JJ, Volker B. Does income inequality have lasting effects on health and trust? Soc Sci Med. 2016;149:37-45. https://doi.org/10.1016/J. SOCSCIMED.2015.11.047.

48. Alvarez E, Kawachi I, Romani JR. Family social capital and health - a systematic review and redirection. Sociol Health IIIn. 2016;39(1):5-29. https:// doi.org/10.1111/1467-9566.12506.

49. Liu B, Floud S, Pirie K, Green J, Peto R, Beral V. Does happiness itself directly affect mortality? The prospective UK million women study. Lancet. 2016; 387(10021):874-881. https://doi.org/10.1016/S0140-6736(15)01087-9.

50. de Souto Barreto P, Rolland Y. Happiness and unhappiness have no direct effect on mortality. 2016. https://doi.org/10.1016/S0140-6736(15)01222-2.

51. Israel Central Bureau of Statistics (ICBS). Social survey: introduction and methodological appendix. Central Bureau of Statistics (Hebrew): Jerusalem; 2017.

52. Lina M. In: Blaise at statistics Netherlands, editor. Hague: Central Bureau of statistic- (Statistics Netherlands); 2003. Retrieved from http://www. blaiseusers.org/2003/papers/Blaise_at_Statistics_Netherlands.pdf.

53. Taber KS. The use of Cronbach's alpha when developing and reporting research instruments in science education. Res Sci Educ. 2018;48(6):1273296. https://doi.org/10.1007/s11165-016-9602-2.

54. United Nations Educational Scientific and Cultural Organization. International Standard Classification of Education ISCED 2011. Montreal: UNESCO Institute for statistics. 2012. Retrieved from http://uis.unesco.org/ sites/default/files/documents/international-standard-classification-ofeducation-isced-2011-en.Pd.

55. Henson RK, Roberts JK. Use of exploratory factor analysis in published research: common errors and some comments on improved practice. Educ Psychol Meas. 2006;66(3):393-416. https://doi.org/10.1177/0013164405282485.
56. Hooper D, Coughlan J, Mullen MR. Structural equation modeling: guidelines for determining model fit. Electron J Bus Res Methods. 2008;6(1):53-60.

57. lacobucci D. Structural equations modeling: fit indices, sample size, and advanced topics. J Consum Psychol. 2010;20(1):90-98. https://doi.org/10. 1016/j.jcps.2009.09.003.

58. Hu LTL, Bentler PM. Cutoff criteria for fit indexes in covariance structure analysis: conventional criteria versus new alternatives. Struct Equ Model Multidiscip J. 1999;6(1):1-55. https://doi.org/10.1080/10705519909540118.

59. Shrout PE, Bolger N. Mediation in experimental and nonexperimental studies: new procedures and recommendations. Psychol Methods. 2002;7(4): 422-45. https://doi.org/10.1037/1082-989X.7.4.422.

60. Preacher KJ, Hayes AF. SPSS and SAS procedures for estimating indirect effects in simple mediation models. Behav Res Methods Instrum Comput. 2004;36(4):717-31. https://doi.org/10.3758/BF03206553.

61. Tofighi D, Mackinnon DP. RMediation: an R package for mediation analysis confidence intervals. Behav Res Methods. 2011;43(3):692-700. https://doi. org/10.3758/s13428-011-0076-X.

62. Bair MJ, Robinson RL, Katon W, Kroenke K. Depression and pain comorbidity: a literature review. Arch Intern Med. 2003;163(20):2433-445. https://doi.org/10.1001/archinte.163.20.2433.

63. Sarid O, Segal-Engelchin D, Cwikel J. The contribution of negative reproductive experiences and chronic medical conditions to depression and pain among Israeli women. Psychology, Health \& Medicine. 2012;17(1):8294. https://doi.org/10.1080/13548506.2011.579987.

\section{Publisher's Note}

Springer Nature remains neutral with regard to jurisdictional claims in published maps and institutional affiliations.

Ready to submit your research? Choose BMC and benefit from:

- fast, convenient online submission

- thorough peer review by experienced researchers in your field

- rapid publication on acceptance

- support for research data, including large and complex data types

- gold Open Access which fosters wider collaboration and increased citations

- maximum visibility for your research: over $100 \mathrm{M}$ website views per year

At $\mathrm{BMC}$, research is always in progress.

Learn more biomedcentral.com/submissions 